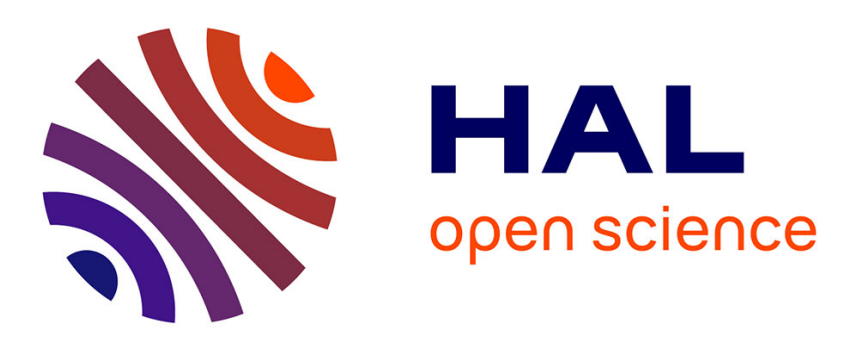

\title{
Dynamics in Clays - Combining Neutron Scattering and Microscopic Simulation
}

\author{
Natalie Malikova, Emmanuelle Dubois, Virginie Marry, Benjamin Rotenberg, \\ Pierre Turq
}

\section{> To cite this version:}

Natalie Malikova, Emmanuelle Dubois, Virginie Marry, Benjamin Rotenberg, Pierre Turq. Dynamics in Clays - Combining Neutron Scattering and Microscopic Simulation. Zeitschrift für Physikalische Chemie, 2010, 224 (1-2), pp.153. 10.1524/zpch.2010.6097 . hal-00531710

\author{
HAL Id: hal-00531710 \\ https://hal.science/hal-00531710
}

Submitted on 22 Nov 2018

HAL is a multi-disciplinary open access archive for the deposit and dissemination of scientific research documents, whether they are published or not. The documents may come from teaching and research institutions in France or abroad, or from public or private research centers.
L'archive ouverte pluridisciplinaire HAL, est destinée au dépôt et à la diffusion de documents scientifiques de niveau recherche, publiés ou non, émanant des établissements d'enseignement et de recherche français ou étrangers, des laboratoires publics ou privés. 


\section{Dynamics in clays - combining neutron scattering and microscopic simulation}

Natalie Malikova: Laboratoire Léon Brillouin, UMR CEA-CNRS 12, CEA Saclay, Gif-sur-Yvette, 91191 , France

Emmanuelle Dubois: Laboratoire de Physicochimie des Electrolytes, Colloides et Sciences Analytiques, UMR-UPMC-CNRS-ESPCI 7195, 4 place Jussieu, 75252 , France

Virginie Marry: Laboratoire de Physicochimie des Electrolytes, Colloides et Sciences Analytiques, UMR-UPMC-CNRS-ESPCI 7195, 4 place Jussieu, 75252 , France

Benjamin Rotenberg: Laboratoire de Physicochimie des Electrolytes, Colloides et Sciences Analytiques, UMR-UPMC-CNRS-ESPCI 7195, 4 place Jussieu, 75252, France

Pierre Turq: Laboratoire de Physicochimie des Electrolytes, Colloides et Sciences Analytiques, UMR-UPMC-CNRS-ESPCI 7195, 4 place Jussieu, 75252 , France 


\begin{abstract}
Mobility of ions and water in clays is at the heart of their remarkable properties of water retention and ion-exchange. It has been addressed here using two microscopic techniques: neutron scattering and molecular dynamics simulations. Neutron scattering gives access exclusively to water dynamics in clays, due to the exceptional sensitivity of neutrons to $\mathrm{H}$ atoms. The data interpretation can be challenging, especially for natural clays such as montmorillonite, with inhomogeneous swelling characteristics. A great improvement is achieved with the use of synthetic materials, as demonstrated here on the case of synthetic (fluoro)hectorite. The standard analytical models for long-range diffusive motion, isotropic translation and its derivative, powder averaged two dimensional translation, have been used to interpret the neutron scattering data. They both agree on the order of magnitude for the diffusion coefficient of water in monohydrated and bihydrated clays, $10^{-10} \mathrm{~m}^{2} \mathrm{~s}^{-1}$ and $10^{-9} \mathrm{~m}^{2} \mathrm{~s}^{-1}$ respectively. While the two-dimensional nature of water diffusion in clays is seen clearly from molecular dynamics simulations, its signature in neutron scattering data is obscured by the powder-averaging of the signal. A novel method, based on a multi-resolution analysis of scattering functions from powder samples, allows never-the-less a clear determination of the dimensionality of water motion in the system. Extracting information on local water motion is difficult on the basis of neutron scattering data only. Various models for localised motion, rotation on a sphere or jump diffusion, have been proposed and used to interpret the observed neutron data, however their applicability is questionable in light of information from molecular dynamics simulations. Aside from aiding the interpretation of neutron scattering data, MD simulations are most valuable in providing information on the behaviour of ions in clays. MD estimates the interlayer ion coefficients as of the some order of magnitude as water, even if the details of ionic motion are strikingly different between the two ions considered here, $\mathrm{Na}^{+}$and $\mathrm{Cs}^{+}$. Further, MD has also allowed to address the topic of ion exchange between clay interlayers and bulk aqueous solution. The microscopic picture of water and ion motion in clays, emerging from neutron scattering and MD simulations, should be treated as a building block of the overall modelling of macroscopic transport in clays, the ultimate property of interest for many clay applications.
\end{abstract}




\section{Introduction}

Clay minerals, rich in silicon and aluminium, are the breakdown products of minerals formed in greater depths of the Earth's crust and which, at the Earth's surface, come into contact with an acidic environment (carbon dioxide dissolved in water), active atmosphere and temperature fluctuations. This so-called weathering process is the main source of sediments and soils on the Earth's surface, on which the biosphere heavily relies. Clays are charged, layered porous materials and give to soil the crucial capabilities of water retention, ion exchange and $\mathrm{pH}$ buffering. A vast number of clay applications has been developed by mankind, beginning with their use in the production of ceramic ware, paper, tiles, damns, in the removal of impurities from air or water, de-colouring, as well as in the field of medicine (treatment of infections) and, understandably, in agriculture (clays act as nutrient / pollutant retention sites). Many modern technologies are also based on clay exploitation, for instance in the field of molecular sieves, catalysis and in the design of disposal sites for radioactive waste $[1,2,3,4,5]$.

In this review, we are concerned with the mobility of water and ions in clays which give rise to their above-mentioned water retention and ion exchange properties. Water and ion mobility on clays has been studied intensely by a variety of techniques, spanning a wide range of length and time scales. The most macroscopic measurements are the tracer experiments, dealing with mobility on the scale of metres and hours or days, e.g. $[6,7,8]$. More mesoscopic/microscopic measurements include those of dielectric spectroscopy, e.g. [9], and nuclear magnetic resonance, e.g. $[10,11]$. Most relevantly for this review, microscopic picture of dynamics in clays has been intensely studied by quasi-elastic neutron scattering studies, e.g. $[12,13,14,15,16]$, and, for the past two decades or so, as summarised in two recent reviews [17, 18], also by microscopic simulations of the type Molecular Dynamics (MD). As in the case of other complex systems, it is very difficult to establish the link between the dynamic/transport information obtained for the very different time and length scales. The complexity of the task arises from the multi-scale structure of the clay system, which presents, at any given scale, a different picture of the porosity in which the ions and water molecules can move. Their motion is possible not only in a two-dimensional microscopic porosity between individual clay layers ( $\AA$ to $\mathrm{nm}$ in size, i.e. the same scale as to the actual size of the water molecules and ions), but also in mesoscopic pores between individual stacks of clay layers (pores of tens of $\mathrm{nm}$ in size or bigger). The short-time behaviour of mobile species in this latter type of pore is similar to diffusion in a bulk solution. On longer time scales, molecules therefore explore several types of pores, and its overall (macroscopic) diffusion coefficient depends on the connectivity between pores, i.e. the geometrical organisation of the porous medium. Quantifying such effects necessarily involves an averaging of properties on a 
given scale in order to define a set of parameters, which are inserted into the larger-scale description $[19,20]$. One such parameter is the so-called tortuosity, defined as the ratio between the distance effectively covered by a tracer through the porous medium and the distance it would have covered following a straight line. The choice of the relevant parameters to describe a given property on larger scales is a key issue, since it implies a loss of information that might exclude some important features from the resulting simplified description. This active field of research, generally referred to as coarse-graining (from microscopic to mesoscopic models) and homogenisation (from the pore scale to the sample scale), is out of the scope here. In this review we concentrate on the dynamics of species in the microscopic porosity of clays. The review is structured in three parts: (1) a reminder of clay structure and hydration, (2) technical aspects of neutron scattering and MD simulations as applied to clays (3) an overview of information on water and ion dynamics in clays obtained by combining the two techniques.

\section{Clay structure and hydration}

Clays are composed of micrometric particles, each of which is a regular stack of negatively charged crystalline alumino-silicate layers (approx. 10100 layers, each $1 \mathrm{~nm}$ thick). The structure of clay layers, depicted in Figure 1 for a montmorillonite clay, has been established from X-ray diffraction studies for almost all types of common clays [5]. The overall negative charge on each layer is compensated by cations such as $\mathrm{Na}^{+}$and $\mathrm{Ca}^{2+}$ inbetween them, in the microscopic porosity called the interlayer. Under increased humidity, the compensating cations become hydrated, the interlayer spacing increases and the system is said to swell, e.g. [21, 22]. This phenomenon and the resulting increased mobility of the compensating ions is at the origin of water retention and ion exchange properties of clays. In general, clay swelling occurs in three stages. It begins in a stepwise manner, called crystalline swelling: discrete layers of water appear in the interlayer, forming the first few hydration shells of the compensating cations. These states are referred to as monolayer/monohydrated, bilayer/bihydrated. Thereafter swelling becomes continuous and in the extreme a colloidal suspension of clay particles ( $<10$ aligned layers) is formed [23, 24, 25, 26]. The stage of crystalline swelling (order of $100 \mathrm{mg}$ of water per $1 \mathrm{~g}$ of clay), is the subject of this review. This region of swelling is of particular interest for the design of disposal sites for radioactive waste, as low-hydrated clays are to act, in a highly compacted form to minimise mesoscopic porosity between clay particles, as barriers for both water and ions between the waste package and the surroundings. This leads us to consider clays with $\mathrm{Cs}^{+}$, a potential radio-nuclide, as the compensating cation, as well as a natural cation, $\mathrm{Na}^{+}$. We consider however non-compacted clays, unlike other studies reported upon in the 
literature, e.g. [27, 15].

The detailed characteristics of crystalline swelling (interlayer spacing as a function of relative humidity) depends critically on the balance between the clay-cation and water-cation interaction, which is itself determined by the nature of the cation together with the magnitude and localisation of the clay charge $[28,5]$. The degree of clay hydration is best characterised using a combination of water adsorption isotherms and X-ray / neutron diffraction. Figure 2 features this type of data for a synthetic Na(fluoro)hectorite (a 2:1 trioctahedral clay), which has exceptionally welldefined swelling characteristics and which shall be discussed throughout this review alongside natural montmorillonite [29]. The monohydrated and bihydrated states for synthetic Na-hectorite appear at around $40 \%$ and $80 \%$ relative humidity and correspond respectively to approximately 4 and 7 water molecules per interlayer $\mathrm{Na}^{+}$ion. This is somewhat lower than the values for natural Na-montmorillonite of a similar charge per unit cell (0.75 e), which are 5-6 and 9-11 water molecules per compensating cation $[30,31]$.

In natural clays, the inhomogeneity in clay layer charge is the principal cause of interstratification (coexistence of different hydration states at a given relative humidity [32]), as well as variable water contents for a given interlayer spacing. This results in a more gradual water uptake seen by water adsorption gravimetry [33] and in broad peaks and irregular spacing in the series of the 00L reflections in diffractograms [34]. A detailed analysis of the irregularity in X-ray diffractograms can be modelled and the proportion of monohydrated, bihydrated etc. layers deduced, for a given relative humidity [34]. Understandably, this inhomogeneity of hydration in natural clays renders the study of water and ions dynamics more difficult than in the case of synthetic clays. However, in light of the applications of clays, which are used almost exclusively in their natural form on the industrial scale, there is certainly the need for parallel studies of model synthetic clays and their natural counterparts.

\section{Microscopic techniques - neutron scatter- ing and molecular dynamics}

Our studies on clays and this review are concerned with dynamics in clays under low levels of hydration, i.e. the crystalline swelling regime. As seen in the previous section, in this regime water is found almost exclusively in the interlayer porosity, where it forms the first few hydration shells of the compensating cations. The dimensions of the interlayer porosity is only of the order of $\AA$ to $\mathrm{nm}$, therefore on the same scale as the actual size of the water molecules and ions. In order to describe the motion of species in this highly confined space, the atomic detail of the diffusing species and of the confining clay layers plays a crucial role. From the need of 
atomic resolution stems the choice of the techniques used - quasi-elastic neutron scattering and molecular dynamics simulations. Both of these techniques give access to the relevant length scale, and allow to probe motion on the scale of ps to ns, which matches very well the timescale of diffusive motion in condensed matter over these nanometric distances. In this section, we introduce some general features of neutron scattering and molecular dynamics as applied to clays. We mention on one hand the practical details of neutron data acquisition and the issues related to the experimental resolution, and discuss briefly the development and testing of model clays and force fields for MD simulations on the other. Finally, we illustrate the nature of the dynamic data obtained by these techniques and link them to analytical models of motion.

In neutron scattering, diffusion of individual atoms or molecules is contained within the incoherently scattered signal. Due to the exceptionally large incoherent neutron scattering cross-section of the ${ }^{1} \mathrm{H}$ isotope of hydrogen, as compared to all other atoms in clays (atoms of $\mathrm{O}, \mathrm{Si}, \mathrm{Al}$ mainly), the incoherently scattered signal from a hydrated clay is dominated by the contribution of water molecules, through their constituent $\mathrm{H}$ atoms. An entire field of neutron scattering, dealing with mobility of H-containing molecules (water and other molecular liquids, organic and biological molecules) confined in all types of matrices (clays, zeolites, metal-organic frameworks, carbon nanotubes etc.), has been built upon the exceptional sensitivity of neutrons to the signal from $\mathrm{H}$ atoms $[35,36,37]$.

The two principal techniques of quasi-elastic neutron scattering are the so-called time-of-flight (TOF) and neutron spin echo (NSE). They measure, respectively, the full scattering function, $S(Q, \omega)$, and the intermediate scattering function, $I(Q, t)$. The respective incoherent contributions, $S_{\text {inc }}(Q, \omega)$ or $I_{\text {inc }}(Q, t)$, which are of interest here, are isolated from the total (coherent and incoherent) signal by a careful choice of the scattering wavevector, i.e. away from peaks of coherent scattering. In theory $S_{i n c}(Q, \omega)$ and $I_{i n c}(Q, t)$ are related by a Fourier transform in time/energy and thus the information they contain is strictly equivalent. Under real experimental conditions, there are several factors that make the comparison between data obtained by different techniques or spectrometers difficult, the first one of which is the experimental resolution. In broad terms, TOF achieves energy resolutions in the range of $10 \mathrm{meV}$ to above $1 \mu \mathrm{eV}$ (corresponding time range: $0.1 \mathrm{ps}$ to $1 \mathrm{~ns}$ ), with the extension of the backscattering (BS) technique (also measuring in the $(Q, \omega)$ domain) below $1 \mu \mathrm{eV}$, therefore equivalently up to 10ns in time. NSE has been conceived as a technique extending the accessible correlation times, its dynamic range is $1 \mathrm{ps}-100 \mathrm{~ns}$ (equivalent energy resolution of $1 \mathrm{meV}$ to $10 \mathrm{neV}$ ). Both TOF and NSE have been used in the past to study dynamics of water in clays. In general, all the initial clay studies dating from the 1980s and early 1990s use TOF [38, 39, 40, 41], while the more recent NSE [42] has been the technique of choice for an increasing number of studies 
throughout the past decade or so $[43,12,13]$. Up to now, dynamics in clays by $\mathrm{BS}$ has been reported upon only once [15]. In general, all the neutron scattering experiments on clays sofar, be it in the time or energy domain, have been used to probe dynamics on a similar time/energy range (approximately 1 ps to $1 \mathrm{~ns}$ ). In other words they have been used under such conditions of resolution to be almost equivalent. As shall be seen, even in this case, the comparison between them is not always trivial.

A brief note is in place on the practicalities of acquiring neutron scattering data on water dynamics in clays by the two main techniques, TOF and NSE. One of the principal differences is the rate of data acquisition, which is significantly higher on TOF than on NSE. This is primarily due to the multi-detector nature of the TOF spectrometers, which allows the measurement of the $S_{\text {inc }}(Q, \omega)$ function at a whole range of scattering angles and thus a range of wavevectors, $Q$, simultaneously. Due to technical issues related to the NSE technique, which relies on the use of polarised neutron beams, the majority of the currently available NSE spectrometers are so-called single-detector spectrometers and thus measurements of $I_{\text {inc }}(Q, t)$ are carried out at a single angle, and thus $Q$, at a time. The second difference is the signal intensity for the case of incoherent scattering as seen by the two techniques. In NSE, the less standard of the two methods, $I_{\text {inc }}(Q, t)$ is measured via the polarisation of the scattered neutron beam. Due to the spin flip of an incoherently scattered neutron (occurring with a probability of $2 / 3$ ), the measured polarisation is thus proportional to $-1 / 3$ of the incoherently scattered intensity [44]. This inherent decrease in the intensity is another reason for the slower data acquisition with NSE. For a few grams of a hydrated clay, our neutron scattering experiments usually take 2-3 days with TOF, though more than 1 week with NSE. These durations apply to the MIBEMOL (TOF) and MUSES (NSE) spectrometers at the Laboratoire Léon Brillouin (Saclay, France), which have incident neutron fluxes of $10^{4}$ neutrons $\mathrm{cm}^{-2} \mathrm{~s}^{-1}$ and $10^{7}$ neutrons $\mathrm{cm}^{-2} \mathrm{~s}^{-1}$ respectively. Detailed clay sample preparation and purification prior to neutron scattering experiments can be found elsewhere, e.g. [13].

While the signal of interlayer water molecules in clays is easily obtained from the incoherently scattered neutron signal, the signal from the other mobile species in the porosity of clays, the compensating counterions (usually the alkali or alkaline Earth metal cations), is negligible. This has lead to many attempts at exchanging the natural cations with ions containing non-exchangeable $\mathrm{H}$ atoms, such as monomethyl- or tetramethylammonium ions and hydrating the system with heavy rather than light water. This should result in a system with $\mathrm{H}$ atoms present only in the ions themselves. Several problems have been encountered on the way however. Apart from possible incomplete exchange of heavy and light water, the major problem stems from the new ions themselves. While still relatively small, they are inevitably larger than the simple inorganic cations and, together with their more complex structure, this is already sufficient to radically change the system, including the swelling properties 
of the clay and of course also the dynamics in the interlayer. Overall, all these ion-exchange attempts have so-far lead to a very limited set of information. If seen at all, such ions appear to be immobile [31].

The need for information on ion mobility in clays leads us to the introduction of the other technique reviewed here in relation to dynamics in clays, classical Molecular Dynamics (MD) simulations. By way of construction, MD simulations give access to the motion of all atoms in a given model system, through real time atomic trajectories [45, 46]. For our purposes, both ion and water dynamics in the clay interlayer is therefore accessible from MD. The crucial and non-trivial part of classical MD is of course the development of the empirical force fields, or interaction potentials, on the basis of which the motion of all atoms in the system is calculated. For a given system, the empirical force fields are tuned to reproduce experimentally available information, thus closing the circle of interdependence between experiments and classical MD. MD simulations on clays appear in the literature ever since the late 1980s. Concurrently, increasingly more accurate interaction potentials for clays have been developed, for the latest refer to [47]. Throughout this review we shall refer mainly to simulations on model clays developed by Skipper et al, in which the interaction force fields were initially tuned to reproduce the experimentally measured adsorption energies of water molecules on a surface of a talc clay $[48,49]$. The model clays used in MD simulations are of course simplified versions of real clays, both in terms of the chemical composition (rare elements cannot be taken into account due to the size of the simulation box) and the regularity of the interlayer spacing (by construction all interlayers are at the same degree of hydration, in contrast to the interstratified natural clays such as montmorillonite). See Figure 3 for the model montmorillonite, referred to in this review. We note that in this particular model, the clay layers and water molecules themselves are treated as rigid. As a first test of the model clay used, it has been shown that the experimentally observed interlayer spacing of monohydrated $\mathrm{Na}$ - and Cs-montmorillonite is reproduced for a water content of 6 water molecules per counterion, while that of bihydrated Namontmorillonite corresponds to 12 water molecules per counterion [50]. (Note that Cs-montmorillonite is considered only in the monohydrated state, the only stable state observed experimentally.) In addition, the experimental hydration energies have been successfully reproduced [51], as well as the interlayer atomic distributions as seen by detailed analysis of high order reflections in X-ray diffractograms [52]. This type of information is also available from neutron diffraction studies [53, 54]. This comparison of static information from simulations and diffraction experiments is necessary, prior to any attempts at exploiting MD to predict the dynamics of the atoms in the clay interlayer.

Having outlined the general strengths and weaknesses of the two techniques used to study microscopic dynamics in clays, we continue with a brief overview of the dynamic quantities measured by neutron scattering 
and MD. The overall approach is to relate these quantities to an analytical model of atomic motion, with the help of which we can characterise the atomic motion using a small set of simple parameters, such as the diffusion coefficients, residence times in preferential sites etc. Schematically, the relationship between the three poles (scattering functions, atomic trajectories and model parameters) is depicted in Figure 4. The connection between atomic trajectories (obtained by MD) and the intermediate scattering function (measured by NSE) can be expressed mathematically as

$$
I_{i n c}(\mathbf{Q}, t)=\frac{1}{N} \sum_{j=1}^{N}\left\langle e^{i \mathbf{Q} \cdot \mathbf{R}_{j}(t)} e^{-i \mathbf{Q} \cdot \mathbf{R}_{j}(0)}\right\rangle
$$

where $\mathbf{R}_{j}(t)$ is the position of scatterer (atom) $j$ at time $t, \mathbf{Q}$ is the wavevector and $N$ is the total number of scatterers in the sample. An additional Fourier transform in time is necessary to arrive at $S_{\text {inc }}(\mathbf{Q}, \omega)$ (quantity measured by TOF). $I_{\text {inc }}(\mathbf{Q}, t)$ is thus a time-correlation function of the positions of individual atoms in the sample, in the reciprocal space of the wavevector. It can be easily calculated from simulated atomic trajectories, $\mathbf{R}_{j}(t)$. (Note that in the vast majority of neutron scattering studies on clays, we deal with powder samples and thus the vector nature of the wave-vector in the measured scattering functions can be ignored.) The rate of decay of $I_{i n c}(Q, t)$, determined with the different techniques (MD or neutron scattering), provides a first assessment of the rate of dynamics seen by each of them. In general, the faster the decay, the faster the motion seen. This type of comparison is presented for water dynamics in clays at the beginning of section 4 .

In order to arrive at an $I(Q, t)$ function from raw NSE and TOF data, for comparison with MD, the effect of experimental resolution has to be eliminated (refer back to Figure 4). For raw NSE data, in $(Q, t)$ form, a simple division is needed. For raw TOF data, in $(Q, \omega)$ form, a deconvolution is necessary, which is not trivial. In practise, we carry out a numerical inverse fourier transform of the raw TOF data and the TOF resolution function, and divide the resulting sets in the $(Q, t)$ domain. This path leads to uncertainties, primarily due to the energy cut-offs in the measured $S_{i n c}(Q, \omega)$, which affect the inverse fourier transform. At the end, the introduction of an arbitrary normalisation parameter is usually necessary to align the height of the TOF data sets in the $(Q, t)$ domain with those of NSE and MD [55, 56, 30].

To extract any more detailed information from the measured dynamic quantities, they have to be interpreted within a well-defined analytical model of motion, which quantifies the characteristic decay or relaxation time of the motion, $\tau$, and ultimately leads to parameters such as the diffusion coefficient. In the language of Figure 4, we need to link the MD trajectories and the neutron scattering functions to the third, remaining pole of analytical models. As MD gives us access to trajectories of atoms in real time and space, which is experimentally unavailable to us, the 
analytical models of motion can be tested on the trajectories in a more direct way. Sometimes viewing the raw trajectories can provide the answer immediately and exclude a certain model.

The development of analytical models of atomic motion starts from the assumption that it is possible to de-couple the different modes of motion (vibrational, rotational and translational) on the basis of their different characteristic time-scales and works within the classical approximation for both momentum and energy transfers [57, 58]. Under these assumptions, the overall $I_{\text {inc }}(Q, t)$ can be factorised into the contributions of each motion type (factorisation in the $(Q, t)$ domain being equivalent to a convolution in the $(Q, \omega)$ domain). The simplest isotropic model for each of these types of motions is vibrations in a harmonic well, free rotation on a sphere and continuous isotropic translation. The exact mathematical expressions for $I_{i n c}(Q, t)$ and $S_{i n c}(Q, \omega)$ for these models can be found elsewhere $[57,58]$. Let us consider their application to water and ion dynamics in clays. For monoatomic species, such as the natural cations in clays considered here, the only relevant model is that of continuous isotropic translation and its derivatives. For water dynamics, we need to consider all three types of motion. Starting with vibrational motion, on the timescale of ps to ns (or equivalently in the region of small, quasielastic energy transfers - meV to $\mu \mathrm{eV}$ ), the effect of this highly energetic motion is not resolved in time and is reduced to a time-independent (but Q-dependent) pre-factor, $e^{-\left\langle u^{2}\right\rangle Q^{2} / 3}$, with $\left\langle u^{2}\right\rangle$ as the sole parameter, the vibrational mean squared displacement [57]. The easiest path for its determination is from the Q-dependence of the scattered intensity integrated over the quasi-elastic energy transfers in the TOF technique. In case of water, this parameter represents primarily the amplitude of librational motion of $\mathrm{H}$ atoms around a hydrogen bond joining two molecules [59]. The effect of librational motion is seen clearly in the current TOF and MD data on water in clays, however not as well in the NSE [60, 61]. For rotational motion of water, the model of rotation on a sphere is often applied. It works well in the case of bulk water, however its applicability to water confined in clays is rather doubtful. This is further discussed in section 4.3. For translational motion of water, we shall discuss, as for the case of ions, the model of continuous isotropic translation (section 4.2) and its derivative, continuous translation under the confinement between two planes (section 4.4). These two models lead, respectively, to the determination of an isotropic diffusion coefficient, $D_{t r}$, and a two-dimensional diffusion coefficient in the plane of the clay layers, $D_{\|}$. It is important to point out a very general difference between translational and rotational type motion. The first type is a long-range diffusive motion, which leads to a dynamic signal all the way to large spatial scales (low Q region) and its relaxation times show a Q-dependence. The second type is a local non-diffusive motion, which leads to a dynamic signal only in the high $\mathrm{Q}$ region (at low $\mathrm{Q}$, it gives rise to a static signal) and the corresponding 


\section{Results: Water and ion dynamics in clays}

After a direct comparison of the simulated and experimentally measured scattering functions obtained for water confined in clay, this section further presents three aspects of modelling of water dynamics in clays: (1) long-range motion within the simplest isotropic model - continuous translation, (2) evidence for local motions such as rotation and jump diffusion, (3) long-range motion within the powder-averaged two-dimensional translational model. In the last part of this session, we describe ion dynamics in clays as seen by MD simulations and finish with a selection of other information on clays obtained by microscopic simulation, such as ion-exchange.

\subsection{Simulated and experimental scattering functions}

Figure 5 gives an example of a direct comparison between the simulated and experimental (NSE) intermediate incoherent scattering functions (from now on referred to simply as $I(Q, t)$ without the subscript for incoherent) for monohydrated Na-montmorillonite. The agreement is good, the same applies to monohydrated Cs-montmorillonite, less so to bihydrated Na-montmorillonite [62, 12]. For the monolayer systems simulation shows a somewhat faster decay (faster dynamics), in the $\mathrm{Na}$ bilayer system the difference reaches a factor of approximately 2 between the experimental and simulated relaxation times. A major factor leading to the disagreement for the bihydrated system is the difference between experimental and simulated water contents and water distribution (interstratification in the natural montmorillonite). Depending on the actual montmorillonite sample and its preparation, the average water content per ion was found to vary between $9-15 \mathrm{H}_{2} \mathrm{O}$ /ion, while the simulated water contents was $12 \mathrm{H}_{2} \mathrm{O}$ /ion. The monohydrated state in the natural clay is better defined. In view of this, we treat the agreement simulation-NSE as satisfactory. Rather than in modifications of interaction potentials for model montmorillonite to reproduce the observed neutron data, further improvement lies primarily in the choice of a better defined experimental system, such as the synthetic hectorite. It is realistic to assume that the corresponding atomic model and interaction potentials for hectorite could be derived from those of montmorillonite.

Apart from the technically straight-forward comparison between the NSE and simulated $I(Q, t)$ data for water confined in clays, a comparison including the TOF data sets, transformed from the $(Q, \omega)$ domain, is presented elsewhere [62]. 


\subsection{Long-range motion - the isotropic diffusion model}

The most basic type of analysis of neutron scattering data on water in clays, and the most common one seen in the literature, is the modelling of the incoherent scattering functions in the low-Q region (usually considered as $Q<1.0 \AA^{-1}$ ) within the isotropic continuous translation, with the translational diffusion coefficient of water, $D_{t r}$, as the outcome. Let us quote the simplest mathematical form with which low- $Q$ scattering functions can be modelled:

$$
\begin{gathered}
I(Q, t)=A \mathrm{e}^{-(t / \tau)}+B \\
S(Q, \omega)_{\text {raw }}=A[\operatorname{Res}(\omega) \otimes \operatorname{Lor}(\omega, 1 / \tau)]+B \operatorname{Res}(\omega)
\end{gathered}
$$

where $\tau$ is the relaxation time, $\operatorname{Lor}(\omega, 1 / \tau)$ stands for a Lorentzian function in $\omega$ with a half width half maximum of $1 / \tau, \operatorname{Res}(\omega)$ is the resolution function in the energy domain. Parameters $A$ and $B$ reflect, to a first approximation, the relative intensities of the mobile and immobile $\mathrm{H}$ atoms in the clay system, which are often used as free parameters, but ultimately need to lead to a reasonable ratio, see later on. Note that data in the $(Q, t)$ domain is interpreted in its resolution-corrected form, which is not the case for the $(Q, \omega)$ domain due to the necessity of de-convolution as discussed earlier. By using the above expressions we assume that in the low- $Q$ region (large length scales) and within the time-scale of the measurement, we probe a single type of relaxation, corresponding to a free isotropic translation. The relaxation time and the translational diffusion coefficient within this model are related via $1 / \tau=D_{t r} Q^{2}[57,58]$. The diffusion coefficient is extracted from the slope of the inverse relaxation times, $1 / \tau$, versus $Q^{2}$, in the limit of small $Q$. For scattering functions measured on real systems however, a few constraints in the above equations need to be lifted, as the simple mono-exponential decay or purely Lorentzian broadening is rarely seen. A very common generalisation is to assume a range of relaxation times, $\tau$. This seems at first sight rather reasonable for a system such as water confined in clays, due to the various water-clay surface interactions. However, the currently available mathematical formalism for dealing with a range of relaxation times is controversial. It relies, in the $(Q, t)$ domain, on the use of a stretched exponential, $I(Q, t)=\mathrm{e}^{-(t / \tau)^{\beta}}$, with the stretch exponent, $\beta$, ranging from 0 to 1 . Apart from the case of $\beta=0.5$ [63], a stretched exponential is not derived from a well-defined analytical model of motion. Moreover, analysis with a stretched exponential can give erroneous results, mostly in the high- $Q$ domain, as shall be discussed in section 4.3. The second generalisation of the above equations is to include a $\mathrm{Q}$-dependence in the parameter $A$. In the $(Q, t)$ domain this is more easily understood by considering that the normalised $I(Q, t)$ function does not depart from 1 , but below this value. 
This is due to a fast relaxation of the type of water librations, which is not resolved properly by the experimental measurement [60].

Figure 6 shows room temperature $I(Q, t)$ data, together with the curves of best fit, for the three montmorillonite systems considered, at a single $Q$ value $\left(1.0 \AA^{-1}\right)$, as well as data at a series of $Q$ for bihydrated synthetic Na-hectorite. While for synthetic hectorite the $I(Q, t)$ can be modelled by a mono-exponential, for the natural montmorillonite it was necessary to use a stretched exponential $[13,62,12]$. This can be accounted for in part by the significantly more homogeneous water distribution in the synthetic clay, as opposed to the natural montmorillonite, as already discussed in section 2 . At the same time, even if a single relaxation is ascribed to the motion of interlayer water molecules in a model clay such as hectorite, some departure from the mono-exponential decay can be explained on the basis of the geometry of the diffusive motion. In other words, the model of isotropic translational diffusion, applied in this most basic analysis, is not the most appropriate. Extensions to a geometrically more applicable model are discussed in section 4.4. Note that the $I(Q, t)$ data for hectorite in Figure 6 descend to zero, as there are no structural $\mathrm{OH}$ groups in this clay. A non-zero background is observed for the montmorillonite clays and compares well with the relative proportion of $\mathrm{H}$ atoms in the structural $\mathrm{OH}$ groups of montmorillonite (static) and those in interlayer water (mobile) [12]. For the corresponding TOF data from the two clay systems, refer to $[13,62,12]$. In general, the data were satisfactorily fitted with a Lorentzian broadening.

Figures 7 feature the extracted inverse relaxation times, $1 / \tau$, plotted versus $Q^{2}$ for the monohydrated and bihydrated synthetic Na-hectorite systems. For analogous data corresponding to natural montmorillonite, refer to $[62,12]$. Table 1 summarises the extracted isotropic translational diffusion coefficients, $D_{t r}$, for both clay types, while both experimental and simulation data are included for the montmorillonite clay. Viewing first the comparison NSE-TOF, a much better agreement is achieved for the hectorite system. This is due to its significantly better and reproducible hydration characteristics. Throughout the course of our investigations it was concluded that the resolution used in the TOF technique $(\mathrm{HWHM}=7.5 \mu \mathrm{eV})$ was insufficient for the determination of diffusion of the monohydrated clays. The analysis predicted incorrect proportions of mobile and immobile $\mathrm{H}$ atoms in the system [12]). Some slow-moving $\mathrm{H}$ atoms were considered simply as static, which lead to an overestimation of the diffusion coefficient extracted. As a general rule, the proportion of $\mathrm{H}$ atoms in these two families (parameters $A$ and $B$ in equations 2 and 3 ) should be determined as accurately as possible from the structure of the clay itself and the amount of water adsorbed, as determined by adsorption mass gravimetry. Modelling of scattering functions very often gives a series of possible results depending on the relative values of $A$ and $B$ and this type of simple, preliminary and independent information can then decide which of these results are indeed plausible. This difficulty is most 
marked in case of the TOF neutron scattering technique, in which ascribing intensity around the zero-energy transfer to either a quasi-elastic or an elastic contribution becomes often impossible to decide, in the absence of other independent information. From our experience, the data in the $(Q, t)$ domain are less likely to suffer from this misinterpretation and we treat the data from NSE as more reliable in this respect. Understandably, the absence of immobile $\mathrm{H}$ atoms in the synthetic hectorite clay simplified greatly the corresponding analysis, especially of TOF data [13].

Referring back to Table 1 and concentrating on the NSE technique, the diffusion coefficients for the montmorillonite and hectorite seem to be very similar, though one must not forget the slightly different hydration values for each system, refer back to section 2 . The last important point regarding Table 1 is the difference between the diffusion coefficient determined from simulation using a direct method (MSD, from particle trajectories) and the indirect method (via the $I(Q, t)$ function and extraction of relaxation times, identical treatment to the neutron data). Remembering that the same particle trajectories are at the beginning of both methods, the differences should be taken as an indication of the errors and distortions introduced during the isotropic averaging of the simulated scattering functions and their subsequent modelling with a stretched exponential [30]. These distortions are non-negligible.

Adding values of isotropic diffusion coefficients from other studies on clays, we can arrive at the following generalisation. The range of diffusion coefficients, at room temperature, for monohydrated clays is $0.5-4$ $\times 10^{-10} \mathrm{~m}^{2} \mathrm{~s}^{-1}$, in other words not reaching above a fourth or a fifth of the bulk water diffusion coefficient. For bihydrated clays, at room temperature, the range becomes $5-10 \times 10^{-10} \mathrm{~m}^{2} \mathrm{~s}^{-1}$, i.e. reaching about half of the bulk water diffusion coefficient. This includes data on three types of clays (hectorite - both natural and synthetic, montmorillonite, vermiculite) and both mono and bivalent counterions. We are doubtful of results suggesting water diffusion very close and even exceeding that of bulk water, as reported in some neutron scattering studies [17] and suspect a problem related to the overestimation of elastic intensity, i.e. proportion of immobile $\mathrm{H}$ atoms, as outlined above.

\subsection{Local motions - rotation and jump diffusion}

The treatment in the previous section was concerned with modelling the scattering functions in the low- $Q$ region, where they are dominated by the signal from the long-range, diffusive motion. When analysing scattering functions in the high- $Q$ region (approximately $Q>1 \AA^{-1}$ ), in other words on the atomic length scale, the details of any localised motions come into play. For water dynamics in clays, we shall mention here two types of localised motion that have been considered in the past studies, more precisely rotation and jump diffusion.

The first type of local motion necessary to model in the high- $Q$ region 
is the rotation of the probed $\mathrm{H}$ atoms around the centre of mass of the water molecule. The model that has been applied for rotational motion of water in clays is the rotation on a sphere [57], which leads to an infinite series of terms in the expression of the scattering functions and in practise only the first two or three of these terms are considered. The combination of this rotational model with a translational diffusion model leads to a first term which contains only the translational motion (important at low Q) and a second combined term, called the trans-rotational term (important at high Q). For more details, the reader is referred to [57, 64, 14]. Rotation on a sphere has been used successfully in the description of the rotational motion of bulk water molecules [57, 59], however it does not describe well the rotational dynamics of water confined in clays. This has been seen in our molecular dynamics simulations and does not seem surprising, in view of the clear orientational structuring of the water molecules with respect to the clay surfaces $[65,66]$. At the same time, a more appropriate model for water reorientation/rotation between two confining (and interacting!) planes is difficult to develop and is currently not available. We have attempted to analyse our data on water in clays using the model of rotation on a sphere combined with translational motion, however the increased number of parameters did not lead to a conclusive set of results.

The jump diffusion model is an extension of the model for free continuous translational diffusion for high- $Q$, or short distances. At this local scale, diffusion is considered to occur by jumps between sites of a mean three-dimensional separation $\left\langle r^{2}\right\rangle$ (various distributions of site separations can be applied, here we shall refer to the commonly used Gaussian distribution) and residence time $\tau_{r e s}$ in each site. This leads to a different relationship between the observed inverse relaxation times versus $Q^{2}: \quad \frac{1}{\tau}=\frac{1}{\tau_{r e s}}\left[1-e^{-Q^{2}\left\langle r^{2}\right\rangle / 6}\right]$. For small $Q$, this model coincides with continuous diffusion, i.e. $1 / \tau=D_{t r} Q^{2}$ with $D_{t r}=\left\langle r^{2}\right\rangle /\left(\tau_{r e s} 6\right)$ [57]. In the high- $Q$ limit, $1 / \tau$ versus $Q^{2}$ tends to an asymptotic limit of $1 / \tau_{\text {res }}$ as opposed to continuing to increase linearly. This departure from linearity at high- $Q$ is taken as the primary evidence for jump diffusion as opposed to continuous diffusion. In the majority of studies on clays using the TOF technique, evidence of a plateau at high $Q$ is commonly seen and the data are thus analysed using the above jump-diffusion model with the Gaussian distribution of jump lengths [41] [40] [38]. Our TOF data also suggest a plateau. Appearance of the plateau has been seen when TOF data are analysed both including and excluding the rotation on a sphere model, in addition to the translational model. The observation of a plateau from the NSE data sets has not been convincing in some studies or completely absent in others $[43,62,12]$. Also modelling the simulated $I(Q, t)$ in our studies with Equation 2 (or the more general stretched exponential) has provided no evidence for this plateau. Even more convincingly, no jump diffusion between well-defined sites was observed in water trajectories, directly available from our MD simulations [66]. (Simulated water tra- 
jectories contrasted greatly with the trajectories of ions in the interlayer, especially for the $\mathrm{Cs}^{+}$ion, which was seen to move by jumps between well-defined sites on the clay surface, as is shown later on in section 4.5.) Similar linear dependence of inverse relaxation times versus $Q^{2}$, without the appearance of a plateau, has been reported in other modelling studies on neighbouring clays [67] [68] which used the simple translational model function at high $Q$.

For bulk water itself, the situation is not entirely clarified. While a plateau at high $Q$ has been observed for water in many quasi-elastic neutron scattering studies, especially for water under super-cooled [69] [70] or confined [71] [72] conditions, simulation data do not reproduce this behaviour [73] [74]. At the same time, slightly different descriptions emerge regarding jump diffusion of water (surface water on zirconium oxide [75]), introducing fixed jump-lengths and thus a slight maximum in the inverse relaxation time versus $\mathrm{Q}^{2}$ curve, before a plateau is reached. We conclude that the currently available evidence for jump diffusion, as opposed to continuous diffusion, for water confined in clays is insufficient. Direct viewing of water trajectories does not show any such behaviour, while indirect evidence via the emergence of a plateau of inverse relaxation times at high $Q$ is not always observed. We note that the basic analysis of scattering functions using a single stretched exponential can lead incidentally to the emergence of this plateau and thus should not be taken by itself as evidence for jump diffusion. We have demonstrated this on the data for a synthetic Na-hectorite [13].

In summary, the interpretation of high- $Q$ data for water confined in clays remains limited, due to the lack of an appropriate model for local rotational motion. Any approximative models, such as the stretched exponential, can lead to erroneous conclusions in the high- $Q$ region, especially regarding jump diffusion.

\subsection{Towards a more appropriate translational model: Diffusion between two parallel planes}

Taking into account the geometry of the clay interlayer, it becomes clear that a two-dimensional translational diffusion model for water is more appropriate than the simple isotropic translational model referred to in the previous sections. This is especially true in the low- $Q$ region, where the length scale probed, approximately $2 \pi / Q$, is greater than the height of the clay interlayer. Simulated trajectories of water molecules in the clay interlayer can be easily projected along the direction perpendicular or parallel to the confining clay layers. From these projections, the effect of confinement is clear, with the mean square displacement of the atoms in the direction perpendicular to the clay layers reaching a constant value at the end of a few ps, which is equivalent to a zero diffusion coefficient in this direction. Diffusive motion is observed only in the plane of the clay layers $[30,66]$. Probing the anisotropy of water motion in clays is therefore 
trivial in the case of simulation, however the experimental equivalent is not easy. It has been tackled in two different ways, depending on the type of the clay system used in experiments.

Some groups have investigated oriented clay samples by neutron scattering, for which the motion parallel and perpendicular to the clay layers can be probed separately by careful orientation of the sample with respect to the incoming neutron beam. The production of oriented clays samples is not a trivial process for clays forming small particles, it can be achieved to within a small angular dispersion $[34,14,39]$. Some experiments have been carried out on a particular type of a vermiculite clay, which forms macroscopic particles $[43,76]$, for which orientation is simple. All neutron scattering data on oriented clay samples show an absence of measurable diffusive motion in the direction perpendicular to the clay layers. We note however that several issues in these studies are not yet sufficiently investigated or explained, such as the choice of only two sample orientations, which seems insufficient to decompose the motion parallel and perpendicular to the clay layers in the wide $Q$-range covered [43, 39], and probably insufficient time/energy range probed, as suggested by the unreasonably high static signal obtained in [14].

While we feel there is a great potential in studies on clays forming macroscopic particles, such as vermiculite, as a natural clay it retains all the disadvantages highlighted previously such as inhomogeneous charge distribution, interstratification and, for large particles, also very slow hydration kinetics. In order to probe anisotropy of water motion in clays, we have therefore chosen an alternative way and concentrate on the synthetic hectorite clay in a non-oriented form, in which the orientation of clay particles is isotropically distributed. If such a system is studied by neutron scattering, the appropriate model is then a continuous translation in a plane (two-dimensional diffusion), but averaged over all possible orientations of this plane, a model referred to as the powder-averaged $2 \mathrm{D}$ model. The corresponding expression of $I(Q, t)$ is well-known,

$$
\left\langle I_{2 d}(Q, t)\right\rangle_{\theta}=\frac{1}{2} \int_{0}^{\pi} e^{-D_{\|} Q^{2}\left[\sin ^{2}(\theta)\right] t} \sin \theta d \theta
$$

where $D_{\|}$is the diffusion coefficient parallel to the clay layers and $\theta$ is the angle between the wave-vector $Q$ and the direction perpendicular to the clay layers [77, 78, 79, 57]. For a direct comparison of the experimental NSE data with this model, it was necessary to eliminate, by rescaling, a short-time decay $(<1 \mathrm{ps})$, corresponding to the previously mentioned librational motion, as seen in our and other MD simulations. The details can be found in ref $[61,13,60]$. Figure 8 shows the resulting rescaled $I(Q, t)$ curves for hectorite bilayer, together with the best fit of the data within the powder averaged two-dimensional model, as well as the simple stretched exponential fit. The data shown are for the low Q region, most appropriate for this purely translational model. As can be seen the powder-averaged 2D model can describe well the observed data with 
the diffusion coefficients in the plane parallel to the clay layers $D_{\|}$of $7.7 \pm 1.0 \times 10^{-10} \mathrm{~m}^{2} \mathrm{~s}^{-1}$. For hectorite monolayer data treated in the same way, refer to [60]. At the same time, the fit with the stretched exponential function (in this $Q$ region a simple exponential gives a similar result) provides just as acceptable a fit of the data. In other words the difference between the two models cannot be made considering the experimental uncertainty. This has been observed for clays ever since the first TOF studies, [38], and is a recurring theme in the analysis of scattering functions for powder systems: the powder averaging obscures the features of a possibly low-dimensional motion. It has been observed in the case of diffusion of surface water on zirconium oxide [75] or in the three-dimensional network of channels of zeolites $[80,35]$.

Overall, in case of water motion in clays, if we aim for a better description of translational motion than what is achieved with a simple exponential (refer back to Figure 6), we come to a choice between the stretched exponential or a powder-averaged 2D model. The first model contains two adjustable parameters, its implementation is simple, however the interpretation can be often misleading. The second model is a one-paramater model with a complex integral form, but has a physical meaning for the system of interest, which in our opinion brings more useful information. We note that the main part (except the tail at long times) of a theoretically generated $I(Q, t)$ data for a powder averaged $2 \mathrm{D}$ model (by definition corresponding to a single relaxation time) can be fitted with a stretched exponential, with a very reasonable relaxation time and the $\beta$ exponent close to 0.7 . This quantifies mathematically the deviation of such a curve from the purely exponential behaviour and shows that a decrease in the $\beta$ exponent can have low-dimensional motion at its origin, not only a range of relaxation times. More on this topic is discussed in $[13,60]$.

Concentrating on the above problem of powder-averaging obscuring the signal from a low-dimensional motion, we have recently used an alternative analysis of the scattering functions measured for water confined in synthetic hectorite. This analysis was carried out for data collected in the $(Q, \omega)$ domain. It is at zero energy transfers, or equivalently the long time tail of an $I(Q, t)$ curve, that a powder averaged 2D (or 1D) motion should feature the most markedly increased intensity in comparison to a signal from an isotropic motion. This is equivalent to some atoms appearing as static, as their motion is being probed perpendicular to the clay layers [81]. Our approach is based on analysing in detail the scattered intensity at zero energy transfers $(\omega=0)$, along with the broadening of the $S(Q, \omega)$ function, collected at a constant $Q$ and at a wide range of energy resolutions. The mathematical relationship between these two quantities follows, for a given shape of the resolution function, a universal Master curve, independent of the diffusion coefficient itself, but strongly dependent on the dimensionality of the motion [82, 81]. Figure 9 summarizes the findings of this type of analysis, where data from water confined in 
synthetic hectorite are compared to bulk water acting as a reference for isotropic motion, for details refer to [81]. Briefly, for each experimentally measured $S(Q, \omega)$, only a set of three values is retained: the half-width half-maximum of the resolution function $(H)$, the wave-vector $(Q)$ and the intensity of the normalised measured scattering function at $\omega=0$, i.e. $S(Q, 0)$. The scattering function is normalised such that its integral over the quasi-elastic region is equal to 1 . After an initial estimate of the diffusion coefficient in $2 \mathrm{D}\left(D_{2 D}\right)$ or isotropic $\left(D_{3 D}\right)$, the quantity $S(Q, 0) \pi D_{n D} Q^{2}$ is plotted versus $\alpha=D_{n D} Q^{2} /\left(D_{n D} Q^{2}+H\right)$. The resulting curve is then compared to the Master curves for a given shape of the resolution function (Gaussian or Lorentzian in most cases) and a given dimensionality of motion. Note that the Master curves are indeed independent of $D_{n D}$ and $Q$ as both $\alpha$ and $S(Q, 0) \pi D_{n D} Q^{2}$ are functions of the ratio $D_{n D} Q^{2} / H$ and thus are dimensionless quantities. The master curve reflects the overall relationship between the maximum and the width of the resolution-broadened scattering function as the experimental resolution increases. A clear divergence is seen in the Master curve corresponding to powder-averaged $2 \mathrm{D}$ motion, as opposed to the saturation in case of isotropic motion. The data for water confined in a bihydrated hectorite clay fall very well on the Master of the 2D motion, with a twodimensional diffusion coefficient of $D_{2 D}=0.7510^{-9} \mathrm{~m}^{2} \mathrm{~s}^{-1}$, in agreement with the previous modelling of the $I(Q, t)$ curves. Overall, this approach provides a clear and convincing determination of the low-dimensionality of motion, it is based on a multi-resolution set of measurements.

The above analysis is based on analysing the signal from mobile scatterers which never-the-less appear static on a given spatial scale, due to their confinement. This is directly linked to the Elastic Incoherent Structure Factor, $\operatorname{EISF}(Q)$, a static quantity measurable by both NSE and TOF, available also from MD, and can lead to the determination of the size of a confining volume [57]. Briefly, $\operatorname{EISF}(Q)$ measures the percentage of scatterers that appear as mobile, as a function of the wavevector (inverse spatial scale). There are however several issues, which make the interpretation of $\operatorname{EISF}(Q)$ difficult. The first is connected to some scatterers appearing as immobile, not due to confinement, but due to insufficient energy/time resolution. This leads to a completely erroneous $\operatorname{EISF}(Q)$, as well as to an overestimation of the dynamics, as already mentioned in section 4.2. Further, for a system such as clays, the confinement is only in one direction and thus its effect is greatly diminished in comparison to a system confinement in all three directions, i.e. spherical pores. All these issues, particularly in connection to clays, are discussed in detail in [12]. 


\subsection{Ion dynamics and other information on clays ob- tained by MD simulations}

As mentioned earlier, neutron scattering is insensitive to the signal from interlayer ions in clays, the path to ion dynamics on the microscopic scale is thus primarily built on MD simulations (and also NMR out of the experimental techniques, though the definition of a spatial scale probed in NMR is much more indirect than in MD or scattering techniques). Having established a reasonable agreement between neutron scattering and MD results on water dynamics in montmorillonite, this gives us confidence in the predictions of the presented MD simulations for this clay, regarding ion dynamics and other types of information. This section features some examples in which MD has been particularly useful in the study of clays.

MD has shown a marked difference between the modes of diffusion for $\mathrm{Na}^{+}$and $\mathrm{Cs}^{+}$ions in montmorillonite. The latter diffuse with a specific site-to-site hopping mechanism along preferential sites on the clay surfaces, as depicted in Figure 10. These sites allow a co-ordination to three oxygen atoms of the adjacent clay layer $[50,66]$. We note that this is also seen in MD on more hydrated pores where the distance between the clay surfaces is large (approx. $35 \AA$ ) and the cation is influenced only by a single surface [83]. There is no evidence for such a behaviour with $\mathrm{Na}^{+}$ counterions, be it in the less (monolayer and bilayer) or more hydrated clays. These ions are more strongly hydrated and hence less influenced by the clay surface. In the Na monolayer state, there is strong evidence for four molecules of water hydrating the $\mathrm{Na}^{+}$ion, at higher hydration states the co-ordination approaches the bulk co-ordination of six [66]. Diffusive motion for ions is seen only in the plane of the clay layers, as in the case of water molecules. Self diffusion coefficients of cations in the monolayer and bilayer montmorillonite are of the order of $10^{-10} \mathrm{~m}^{2} \mathrm{~s}^{-1}$ and $10^{-9}$ $\mathrm{m}^{2} \mathrm{~s}^{-1}$, respectively, therefore of the same order of magnitude as interlayer water $[50,66]$. The ion diffusion coefficient in bihydrated states is approximately $80 \%$ of the bulk one for the sodium ion and $50 \%$ for the cesium ion [50]. It is again an indication that $\mathrm{Na}^{+}$are less influenced by the presence of the clay surface. The temperature dependence of these diffusion coefficients has been also investigated [66].

More details on water motion have also been obtained from MD simulation on the highly hydrated clays (interlayer height approx. $35 \AA$ ), differentiating between water molecules linked to the clay surface by hydrogen bonds and the remaining ones. It has been shown that $\mathrm{H}$-bond formation and breaking with the oxygen atoms of clay surfaces are respectively faster and slower than the same processes between water molecules in bulk solutions [83]. This suggests a stronger hydrogen bonding with clay surfaces, probably because of the presence of an electric field, originating from the charge of the clay surface, which orients the water dipoles towards it. Simulations can also distinguish between water molecules close to the surface and water molecules in the middle of the highly hydrated 
pore, and quantify the diffusion coefficient as a function of the distance from the clay surface. This is not possible with the neutron scattering techniques used, which are bulk techniques and would inevitably measure average properties of all water molecules in the system (the residence time of mobile species near the surface does not exceed a few picoseconds). As expected, diffusion coefficients along the surfaces are lower than for the bulk, they are close to those obtained in bihydrated states. This suggests that the presence of another clay surface or layer of water seems irrelevant already beyond a few layers of water. A single clay layer influences the adjacent solution in its structure and dynamics parallel to the surface only up to $10 \AA$. For dynamics perpendicular to the surface, the effect extends to distances larger than $20 \AA[83]$.

Beyond the behaviour of water and ions inside the clay interlayer, we have also been interested by the exchange of ions (both positive and negative) between the clay interlayer and a more mesoscopic pore filled with an aqueous solution, e.g. pores between individual clay particles. We have computed the rates of exchange between the two types of pores [84, 85]. They are fast for water and cations and much slower for anions, which are excluded from the interlayer spaces because of their negative charge. These exchange rates, like the diffusion coefficients, can be used as input parameters in macroscopic transport models of clays, which take into account the global geometry of the clay sample. Molecular simulations have also clarified the microscopic origin of ion exchange selectivity in clays: It is well known experimentally that the exchange of the natural $\mathrm{Na}^{+}$ counterion with larger alkaline ions such as $\mathrm{Cs}^{+}$is thermodynamically favourable. Simulations allowed to demonstrate, in conjunction with experimental thermodynamic data, that the driving force for this exchange is not found in specific, favourable interactions between $\mathrm{Cs}^{+}$, as was thought previously, but in the higher hydration energy of the $\mathrm{Na}^{+}$ions released into the solution $[86,87]$.

\section{Conclusion and Perspectives}

In this review we have shown the breadth of information that can be obtained regarding dynamics of simple ions and water in the interlayer of clays using a combination of neutron scattering and MD simulations. Natural clays are usually highly inhomogeneous materials and studies on synthetic counterparts play a very significant role in understanding complex experimental data, such as scattering functions. Even for synthetic clays, the analysis of neutron scattering data requires a prior detailed characterisation of the system, especially in terms of water content and a careful consideration of the available experimental resolution. In the literature we see a general consensus regarding the measured water (translational) diffusion coefficients in clays. As far as details on local water motion are concerned, a clear agreement has not been reached. There is a 
lack of appropriate analytical models, such as those for rotational motion. A faithful model has to be consistent with the data from the scattering functions, but also with information from the real space, i.e. particle trajectories available from $\mathrm{MD}$, which is an independent route to its testing. Ionic motion in clays as seen by MD shows very distinct types of motion for different cations. This has to be compared with experimental data, such as NMR, though the link to the measured quantity is not as straight forward as in the case of scattering functions.

Beside the simple ions and water, other species in the clay interlayer have been increasingly studied, such as small hydrocarbons or other organic molecules. This is indeed an important issue considering the role of clays in the soil as a reservoir of organic nutrients or pollutants. It is of concern even to other clay applications, such as to the stability of wells drilled through clay sediments for the extraction of crude oil. Even in the scenario of radioactive waste disposal, it is thought that heavy radioactive ions can escape from the waste package through the clay barrier by binding to organic molecules. Aside from organic species, the suggestion of storing carbon dioxide in former underground reservoirs of crude oil, has incited studies into the behaviour of this gas in the clay interlayer. As mentioned earlier, the dynamics of species in the clay interlayer as reviewed here cannot provide directly the answer to the above issues. This microscopic description is a necessary building block, to be inserted into a more macroscopic model of transport through the multiple porosity of the clay material.

\section{Acknowledgments}

We are indebted to Josef Breu for providing samples of synthetic hectorite and Laurent Michot and Manuel Pelletier for mass adsorption gravimetry measurements. We are also very grateful to Gilles Andre, Jean-Marc Zanotti, Stephane Longeville and Jacques Ollivier for valuable help throughout neutron scattering experiments. 


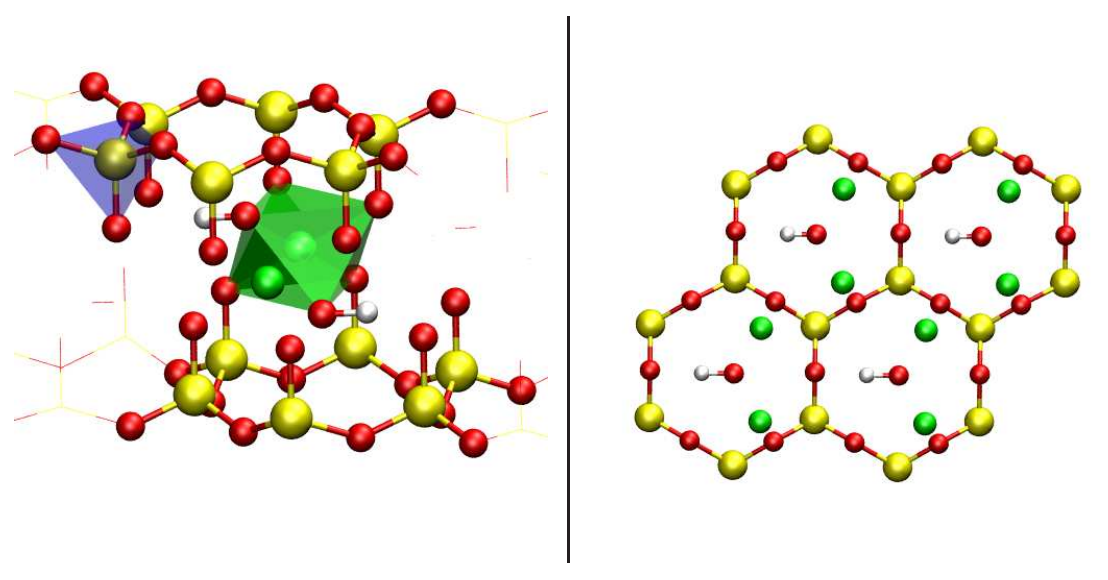

Figure 1: Structure of a montmorillonite (2:1 dioctahedral clay) layer. The side (left) and top (right) view are shown (green: Al, yellow: Si, red: $\mathrm{O}$, white: $\mathrm{H}$ atoms). A central sheet of octahedra of $\mathrm{Al}^{3+}$ oxide (in green) is fused on each side with a sheet of tetrahedra of $\mathrm{Si}^{4+}$ oxide (in purple). From the top, the layer presents a network of hexagons made of Si and $\mathrm{O}$ atoms, with an $\mathrm{OH}$ group (part of an $\mathrm{Al}$ octahedron) buried in its centre (also called the hexagonal cavity). The overall negative charge on clay layers arises from the substitution of $\mathrm{Al}^{3+}$ or $\mathrm{Si}^{4+}$ with lower valence cations.

\begin{tabular}{|c||cccc|}
\hline $\mathrm{D}_{t r}^{\text {water }} \times 10^{-10} \mathrm{~m}^{2} \mathrm{~s}^{-1}$ & NSE & TOF & Sim $^{\text {indirect }}$ & Sim $^{\text {direct }}$ \\
\hline \hline Na-monolayer Mte & 2.5 & $(8.0)$ & 2.5 & 3.8 \\
\hline Cs-monolayer Mte & 1.5 & $(11.0)$ & 1.5 & 2.8 \\
\hline Na-bilayer Mte & 5.0 & 10.0 & 10.0 & 8.1 \\
\hline Na-monolayer Hte & 1.5 & - & - & - \\
\hline Na-bilayer Hte & 4.6 & 4.3 & - & - \\
\hline
\end{tabular}

Table 1: Isotropic diffusion coefficients $\left(\mathrm{D}_{t r}\right)$ of water for the montmorillonite (Mte) and synthetic hectorite (Hte) clay studied. Sim ${ }^{\text {indirect }}$ refers to simulated diffusion coefficients determined via the calculation of the $I(Q, t)$ function and subsequent fitting with stretched exponential decay. $\mathrm{Sim}^{\text {direct }}$ refers to diffusion coefficients determined directly from the simulated trajectories via the MSD method. Data refers to room temperature. The error is $\pm 0.1-0.3 \times 10^{-10} \mathrm{~m}^{2} \mathrm{~s}^{-1}$. 

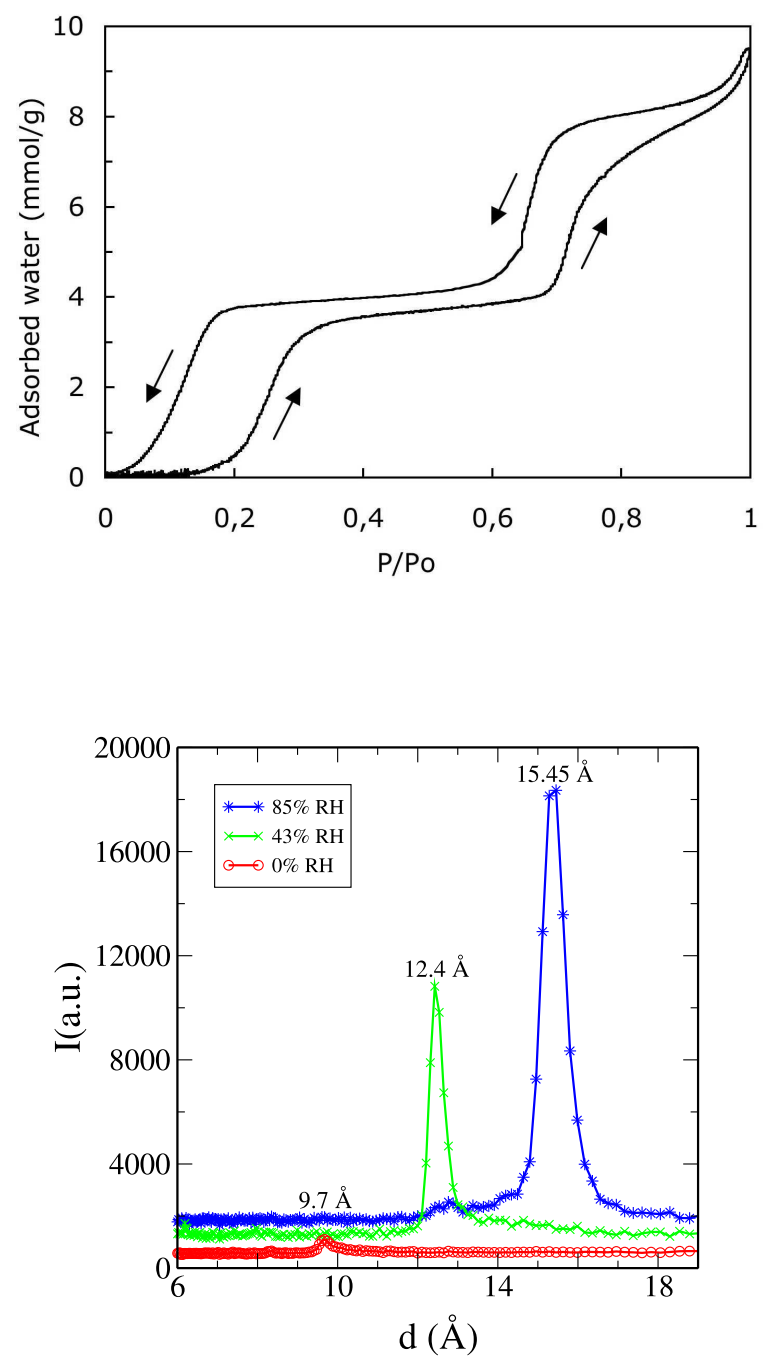

Figure 2: Swelling characteristics of synthetic Na-(fluoro)hectorite, as measured by water adsorption isotherm (top) and neutron diffraction (bottom), both at room temperature. We correlate the weight of water adsorbed at a given relative humidity $\left(\mathrm{P} / \mathrm{P}_{0}\right.$, with $\mathrm{P}_{0}$ the saturated water vapour pressure) and the interlayer spacing (d) seen from the 001 reflection in the diffractogram. For further details see ref [13]. The (fluoro)hectorite unit cell is $\left[\mathrm{Na}_{\mathrm{x}}\right]^{\text {inter }}\left[\mathrm{Mg}_{6-\mathrm{x}} \mathrm{Li}_{\mathrm{x}}\right]^{\text {oct }}\left[\mathrm{Si}_{8}\right]^{\text {tet }} \mathrm{O}_{20} \mathrm{~F}_{4}$ with $\mathrm{x}=0.8$. Charge per unit cell is $0.8 \mathrm{e}$ and $\mathrm{Na}^{+}$is the interlayer cation. The material is fluorated, i.e. the $\mathrm{OH}^{-}$groups in the octahedral sheet of the clay are replaced by $\mathrm{F}^{-}$atoms. Figure reprinted with permission from Journal of Physical Chemistry C, ref [13]. Copyright 2007 American Chemical Society. 


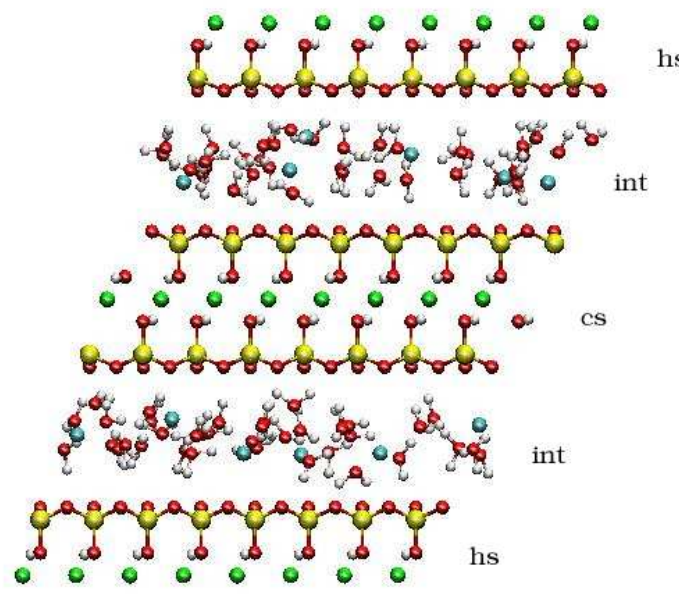

Figure 3: Simulation box of a model montmorillonite clay (unit cell: $\left.\mathrm{Na}_{0.75}\left[\mathrm{Si}_{8}\right]\left(\mathrm{Al}_{3.25} \mathrm{Mg}_{0.75}\right) \mathrm{O}_{20}(\mathrm{OH})_{4}\right)$ for Molecular Dynamics simulations - for colour code refer to the legend of Figure 1. It consists of two halflayers (hs), central layer (cs) and two interlayer spaces (int) with numbers of ions, $\mathrm{Na}^{+}$or $\mathrm{Cs}^{+}$, and water molecules in a predetermined ratio, corresponding to a given hydration state. Periodic boundary conditions applied in all three directions result in a model clay system consisting of infinite parallel equally-spaced clay layers. The inter-atomic pair potentials are the Lennard-Jones and Coulombic potentials representing the Van der Waals/steric repulsion and electrostatic interactions respectively. Atomic charges and Lennard Jones parameters for each atom were taken from Smith et al [88] for the clay and Berendsen et al [89] for the model of water (SPC/E model). Further details of these MD simulations can be found in refs $[62,12,66]$. Figure reprinted from Chemical Physics 2005, ref [62], with permission from Elsevier. 


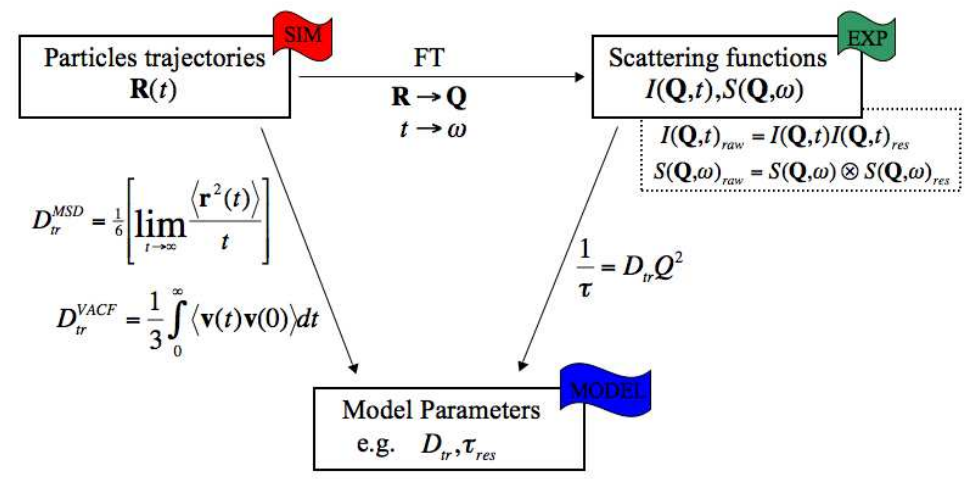

Figure 4: Overview of the dynamic information available from MD and neutron scattering and their link to analytical models and parameters. Particle trajectories and scattering functions are related via a timecorrelation relation and Fourier Transforms (FT). Within a proposed analytical model of motion, we can determine model parameters from MD and neutron data. Using the example of the model for continuous translational diffusion (based on random walk), the translational diffusion coefficient, $D_{t r}$, is calculated from MD trajectories using the mean square displacement (MSD) or the velocity auto-correlation (VACF) relations. For the scattering functions, $D_{t r}$ is determined via the characteristic decay or relaxation time, $\tau$.

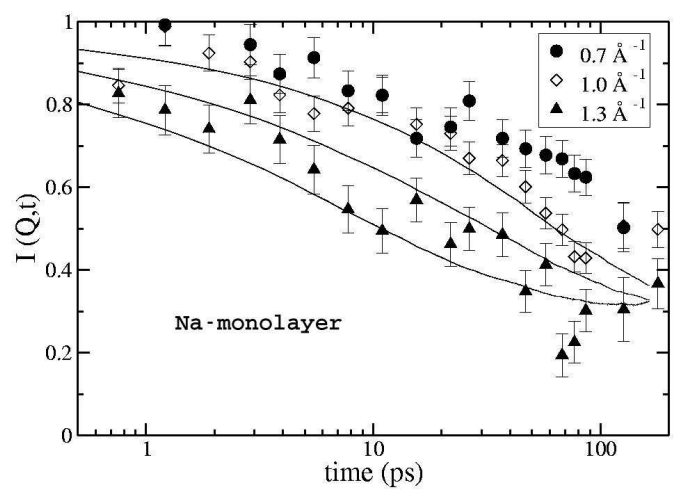

Figure 5: Intermediate scattering functions, $I(Q, t)$, from simulation (full lines) and NSE experiments (symbols) for monohydrated Namontmorillonite at room temperature. Figure reprinted with permission from Journal of Physical Chemistry B, ref [12]. Copyright 2006 American Chemical Society. 

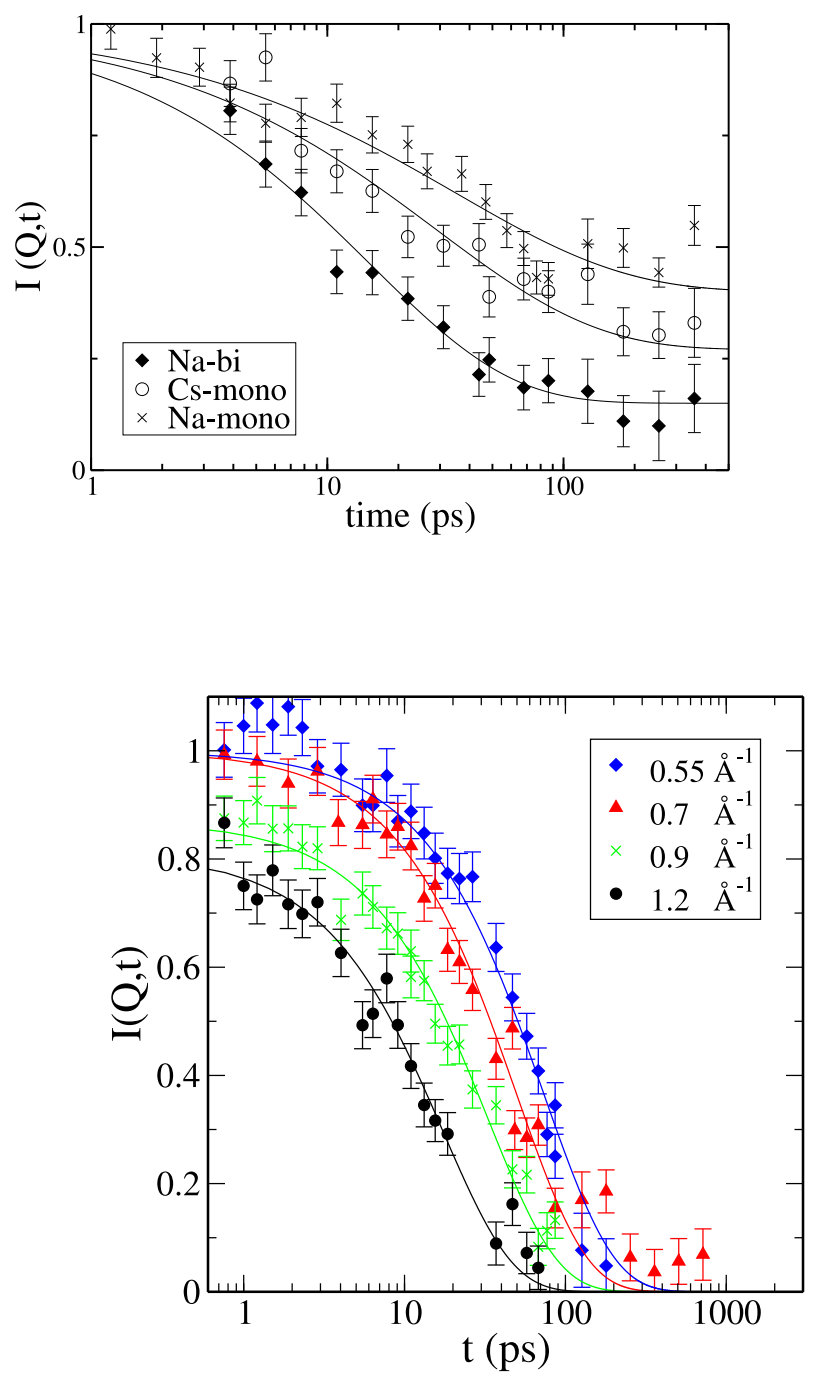

Figure 6: Intermediate scattering function $I(Q, t)$ at a single $Q\left(1.0 \AA^{-1}\right)$ for three natural montmorillonite systems (top), $I(Q, t)$ data for bihydrated Na-hectorite at a series of $Q$ values (bottom). All data refer to room temperature. The lines of best fit shown are stretched exponentials for montmorillonite data and mono-exponentials for hectorite data. Figure reprinted in part with permission from Journal of Physical Chemistry C, ref [13]. Copyright 2007 American Chemical Society. 


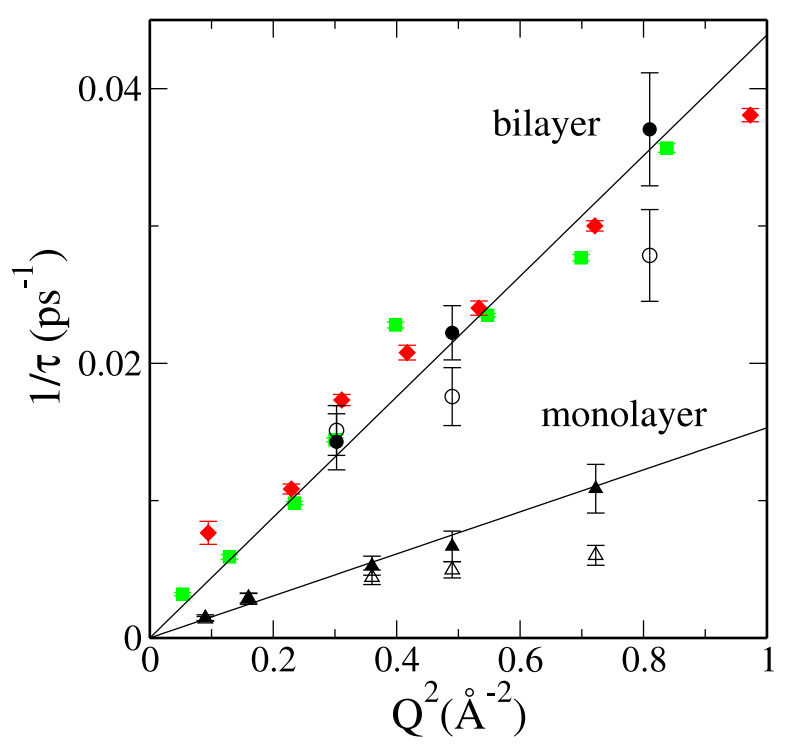

Figure 7: Inverse relaxation time, $1 / \tau$ or $1 /\langle\tau\rangle$, versus $Q^{2}$ for monolayer and bilayer of Na-hectorite at room temperature. Monolayer: black triangles (NSE). Bilayer: black circles (NSE), green squares (TOF, high resolution), red diamonds (TOF, medium resolution). Full symbols - data from mono-exponential analysis, empty symbols - data from stretched exponential analysis. Figure reprinted with permission from Journal of Physical Chemistry C, ref [13]. Copyright 2007 American Chemical Society. 


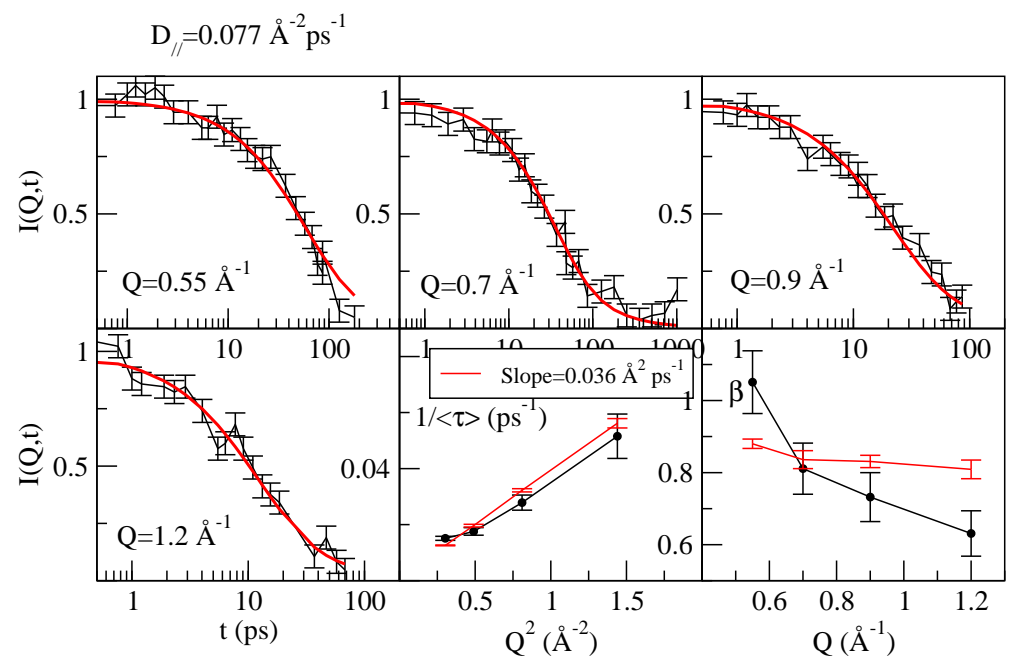

Figure 8: Experimental $I(Q, t)$ curves from NSE for bilayer of Na-hectorite (black data points) with the corresponding isotropic (stretched exponential) fits (black line) and curves generated using a powder averaged 2D model with $D_{\|}=7.7 \times 10^{-10} \mathrm{~m}^{2} \mathrm{~s}^{-1}$ and $D_{\perp}=0$ (red lines). Inverse average relaxation times, $1 /\langle\tau\rangle$, and stretch exponent, $\beta$, as a function of $Q$ from a fit of the experimental data points and the curves generated using the powder averaged 2D model with a single stretched exponential (black circles and red plus respectively). Data refers to room temperature. Figure reprinted from Journal of Physics - Condensed Matter 2008, ref [60], with permission from IOP Publishing Ltd. 


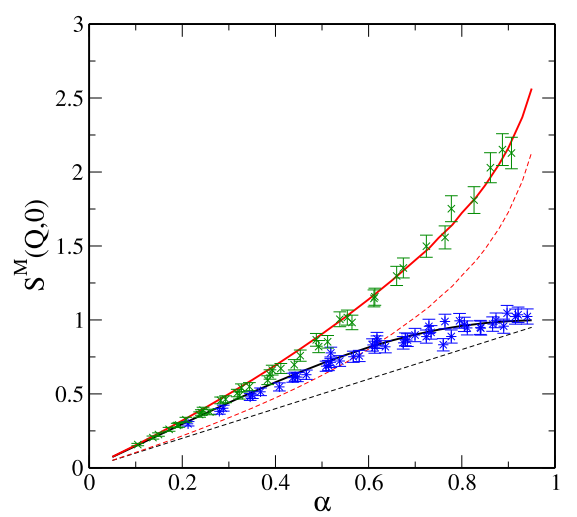

Figure 9: $S^{M}(Q, 0)\left(=S(Q, 0) \pi D_{n D} Q^{2}\right)$ versus $\alpha\left(=D_{n D} Q^{2} /\left(D_{n D} Q^{2}+\right.\right.$ $H)$ ), for $3 \mathrm{D}$ (isotropic) and powder-averaged 2D motion, where $D_{n D}$ denotes the diffusion coefficient for n-dimensional motion. For 3D motion, we show the theoretical prediction (master curve) in case of Gaussian resolution (black line) and a corresponding experimental data set collected for bulk water (blue $*$ ). For powder-averaged 2D motion, the master curve in case of Gaussian resolution (red line) is accompanied by a set of experimental data for water confined in a synthetic clay (green $\times$ ). The analogous master curves in case of $3 \mathrm{D}$ and powder-averaged $2 \mathrm{D}$ signal combined with Lorentzian resolution are shown as dashed lines. All experimental data refer to room temperature. Figure reprinted from Physical Review Letters, ref [81]. Copyright 2008 by the American Physical Society. 


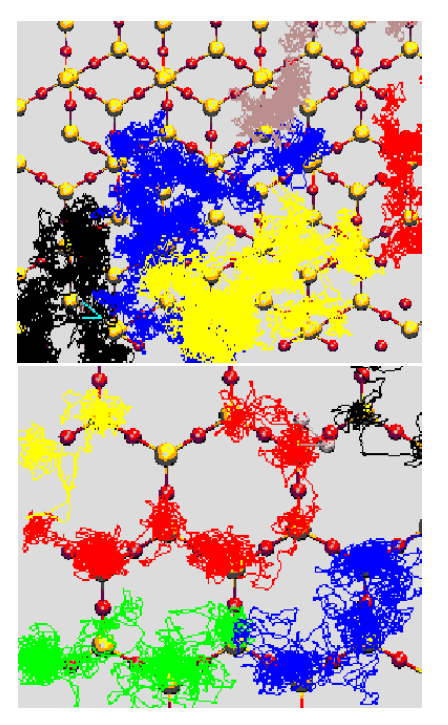

Figure 10: Simulated trajectories of $\mathrm{Na}^{+}$(top) and $\mathrm{Cs}^{+}$(bottom) ions in monohydrated montmorillonite, projected onto the plane of the clay layers. In this projection, each clay layer presents a network of hexagons formed of Si (yellow) and O (red) atoms. The trajectories correspond to a temperature of approximately 400K. The same mode of diffusion for each ion is observed at room temperature: site-to-site jumps for $\mathrm{Cs}^{+}$and more diffuse motion for $\mathrm{Na}^{+}[66]$. 


\section{References}

[1] L. Fowden, R. M. Barrer, P. B. Tinker - editors. Clay Minerals: Their structure, behaviour and use; Royal Society - London, 1984.

[2] B. Velde. Introduction to clay minerals; Chapman and Hall, 1992.

[3] R.F. Giese, C.J. van Oss. In Surfactant science series, Vol. 105; Marcel Dekker, 2002.

[4] ANDRA. Les Recherche de l'ANDRA sur le stockage géologique des déchets radioactifs à haute activité et à vie longue; www.andra.fr, 2005 .

[5] ed. F. Bergaya, B. K. G. Theng, G. Lagaly. Handbook of clay science; Elsevier, 2006.

[6] M. Molera, T. Eriksen. Radio. Acta 2004, 90, 753-760.

[7] C. Daqing, T. Eriksen. Radio. Acta 1998, 82, 287-292.

[8] T. Kozaki, K. Inada, S. Sato, H. Ohashi. J. Contamin. Hydrology 2001, 47, 159-170.

[9] Rotenberg, B.; Cadene, A.; Dufrêche, J.-F.; Durand-Vidal, S.; Badot, J.-C.; Turq, P. J. Phys. Chem. B 2005, 109(32), 15548-15557.

[10] A. Delville, M. Letellier. Langmuir 1995, 11, 1361-1367.

[11] P. Porion and L. J. Michot and A. M. Faugère and A. Delville. J. Phys. Chem. C 2007, 111(14), 5441-5453.

[12] N. Malikova and A. Cadène and V. Marry and E. Dubois and P. Turq. J. Phys. Chem. B 2006, 110, 3206-3214.

[13] N. Malikova, A. Cadène, E. Dubois, V.Marry, S.Durand-Vidal, P. Turq, J.Breu, S.Longeville and J-M.Zanotti. J. Phys. Chem. C 2007, 111, 17603-17611.

[14] L. J. Michot and A. Delville and B. Humbert and M. Plazanet and P. Levitz. J. Phys. Chem. C 2007, 111, 9818-9831.

[15] F. G. Sanchez, F. Juranyi, T. Gimmi, L. Van Loon, T. Unruh, L.W. Diamond. J. Chem. Phys. 2008, 129(17), 174706.

[16] H. N. Bordallo, L. P. Aldridge, G. J. Churchman, W. P. Gates, M. T. F. Telling, K. Jiefer, P. Fouquet, T. Seydel, S. A. J. Kimber. J. Phys. Chem. C 2008, 112(36), 13982-13991.

[17] N. T. Skipper and P. A. Lock and J. O. Titiloye and J. Swenson and Z. A. Mirza and W. S. Howells and F. Fernandez-Alonso. Chem. Geol. 2006, 230, 182-196. 
[18] D. Bougeard and K. S. Smirnov. Phys. Chem. Chem. Phys. 2007, 9, 226-245.

[19] Whitaker, S. The Method of Volume Averaging; Kluwer Academic Publisher, 1999.

[20] Torquato, S. Random Heterogeneous Materials : Microstructure and Macroscopic Properties; Springer Verlag, 2001.

[21] K. Norrish. Disc. Faraday Soc. 1954, 18, 120-134.

[22] G. Sposito, R. Prost. Chemical Reviews 1982, 82, 553-573.

[23] L.L. Schramm, J.C.T. Kwak. Clays and Clay Minerals 1982, 30, 40-48.

[24] I. Bérend, J.M. Cases, M. Francois, J.P. Uriot, L.J. Michot, A. Masion, F. Thomas. Clays and Clay Minerals 1995, 43, 324-336.

[25] J.M. Cases, I. Bérend, M. Francois, J.P. Uriot, F. Thomas, J. E. Poirier. Langmuir 1992, 8, 2730-2739.

[26] J.M. Cases, I. Bérend, M. Francois, J.P. Uriot, L.J. Michot, F. Thomas. Clays and Clay Minerals 1997, 45, 8-22.

[27] T. Kozaki, H. Sato, S. Sato, H. Ohashi. Engineering Geol. 1999, 54, 223-230.

[28] Hensen, E. J. M.; Smit, B. J. Phys. Chem. B 2002, 106, 12664-12667.

[29] Breu, J.; Seidl, W.; Stoll, A.; Lange, K.; Probst, T. Chem. Mater. 2001, 13, 4213-4220.

[30] N. Malikova. Dynamique de l'eau et des ions dans les argiles de type montmorillonite par simulation microscopique et diffusion quasiélastique des neutrons $\mathrm{PhD}$ thesis, Université Pierre et Marie Curie (Paris 6),http://tel.archives-ouvertes.fr/, 2005.

[31] A. Cadène. Etude expérimentale multiéchelle du transport ionique et aqueux en milieu poreux chargé: argile $\mathrm{PhD}$ thesis, Université Pierre et Marie Curie (Paris 6), 2005.

[32] C.E. Weaver. Am. Mineralogist 1956, 41, 202-221.

[33] L. J. Michot, F. Villiéras, M. François, I. Bihannic, M. Pelletier, J.-M. Cases. C. R. Geoscience 2002, 334, 611-631.

[34] Ferrage, E.; Lanson, B.; Sakharov, B.A.; Drits, V.A.. Am. Mineralogist 2005, 90, 1358-1374.

[35] H. Jobic, D. N. T. Microporous and Mesoporous Materials 2007, 102, 21-50. 
[36] Kolesnikov, A. I.; Zanotti, J.-M.; Loong, C.-K.; Thiyagarajan, P.; Moravsky, A. P.; Loutfy, R. O.; Burnham, C. J. Phys. Rev. Lett. 2004, 93(3), 035503.

[37] Salles, F.; Jobic, H.; Maurin, G.; Koza, M. M.; Llewellyn, P. L.; Devic, T.; Serre, C.; Ferey, G. Phys. Rev. Lett. 2008, 100, 245901.

[38] D.J. Cebula, R.K. Thomas, J.W. White. Clays and Clay Minerals 1981, 29, 241-248.

[39] C. Poinsignon, J. Estrade-Szwarckopf, J. Conard, A.J. Dianoux. Proc. Int. Clay Conf., Denver 1985 1987, pages 284-291.

[40] J.J. Tuck, P.L. Hall, M.H.B. Hayes, D.K. Ross, J.B. Hayter. J.Chem.Soc., Faraday Trans. 1 1985, 81, 833-846.

[41] J.J. Tuck, P.L. Hall, M.H.B. Hayes, D.K. Ross, C. Poinsignon. J.Chem.Soc., Faraday Trans. 1 1984, 80, 309-324.

[42] F. Mezei. Z. Physik 1972, 255, 146-160.

[43] J. Swenson and R. Bergman and S. Longeville. J. Chem. Phys. 2001, 115, 11299-11305.

[44] G. L. Squires. Introduction to the theory of Thermal Neutron Scattering; Dover Publications, 1996.

[45] D. Frenkel, B. Smit. Understanding Molecular Simulations, From Algorithms to Applications; Academic Press, 2002.

[46] M.P. Allen, D.J.Tidelsley. Computer simulation of Liquids; Oxford Science Publications, 2002.

[47] R. T. Cygan, J.-J. Liang, A. G. Kalinichev. J.Phys.Chem B 2004, 108, 1255-1266.

[48] N.T. Skipper, K. Refson, J.D.C. McConnell. Clay Minerals 1989, 24, 411-425.

[49] N.T. Skipper, K. Refson, J.D.C. McConnell. J. Chem. Phys. 1991, 94(11), 7434-7445.

[50] V. Marry, P. Turq. J.Phys.Chem. B 2003, 107, 1832-1839.

[51] V. Marry, P. Turq, T. Cartailler, D. Levesque. J.Chem.Phys. 2002, $117,3454-3463$.

[52] Ferrage, E.; Lanson, B.; Malikova, N.; Plancon, A.; Sakharov, B.A.; Drits, V.A.. Chem. Mater. 2005, 17, 3499-3512.

[53] N.T. Skipper, A. K. Soper, M. V. Smalley. J. Phys. Chem. 1994, 98(3), 942-945. 
[54] D. H. Powell, K. Tongkhao, S. J. Kennedy, P. G. Slade. Clays and Clay Minerals 1997, 45(2), 290-294.

[55] F. Trouw, D. Bedrov, O. Borodin, G. D. Smith. Chem. Phys. 2000, 261, 137-148.

[56] W. S. Howells. Physica B 1996, 226, 778-81.

[57] M. Bée. Quasi-elastic neutron scattering; Principles and Applications in Solid State Chemistry, Biology and Material Science; Adam Hilger, 1988.

[58] R. Hempelmann. Quasielastic neutron scattering and solid state diffusion; Clarendon Press, Oxford, 2000.

[59] J. Teixeira, M.-C. Bellissent-Funel, S. H. Chen, A. J. Dianoux. Phys. Rev. A 1985, 31(3), 1913-1917.

[60] V. Marry, N. Malikova, A. Cadène, E. Dubois, S. Durand-Vidal, P. Turq, J. Breu, S. Longeville and J.-M. Zanotti. J.Phys.: Condens. Matter 2008, 20, 10425.

[61] S.-H. Chen and C. Liao and F. Sciortino and P. Gallo and P. Tartaglia. Phys. Rev. E 1999, 59(6), 6708-6714.

[62] N. Malikova and A. Cadène and V. Marry and E. Dubois and P. Turq and J.-M. Zanotti and S. Longeville. Chem. Phys. 2005, 317, 226-235.

[63] W. Götze and L. Sjögren. Rep. Prog. Phys. 1992, 55, 241-376.

[64] L. Liu and A. Faraone and S.-H. Chen. Phys. Rev. E 2002, 65, 041506.

[65] F.C. Chang, N.T. Skipper, G. Sposito. Langmuir 1995, 11, 27342741.

[66] N. Malikova, V. Marry, J.-F. Dufrêche, C. Simon, P. Turq and E. Giffaut. Mol.Phys. 2004, 102, 1965-1977.

[67] M. Arab, D. Bourgeard, K. S. Smirnov. Phys. Chem. Chem. Phys. 2003, 5, 4699-4707.

[68] M. Arab, D. Bourgeard, K. S. Smirnov. Phys. Chem. Chem. Phys. 2004, 6, 2446-2453.

[69] J. Teixeira, M.-C. Bellissent-Funel, S.-H. Chen, A. J. Dianoux. J. de Phys. 1984, C7, 65-71.

[70] M.-C. Bellissent-Funel, J. Teixeira. J. Mol. Struc. 1991, 250, 213230. 
[71] M.-C. Bellissent-Funel, K. F. Bradley, S. H. Chen, J. Lal, J. Teixeira. Physica A 1993, 201, 277-285.

[72] J. Teixeira, J.-M. Zanotti, M.-C. Bellissent-Funel, S. H. Chen. Physica B 1997, 234-236, 370-374.

[73] J. J. Ullo. Phys. Rev. A 1987, 36(2), 816-826.

[74] S.-H. Chen, P. Gallo, F. Sciortino, P. Tartaglia. Phys. Rev. E 1997, $56(4), 4231-4243$.

[75] E. Mamontov. J. Chem. Phys. 2004, 121(18), 9087-9097.

[76] J. Swenson and R. Bergman and W.S. Howells. J. Chem. Phys. 2000, 113, 2873-2879.

[77] P. L. Hall, D. K. R. Mol. Phys. 1978, 36(5), 1549-1554.

[78] P.L. Hall, D.K. Ross. Mol. Phys. 1981, 42, 673-682.

[79] A. J. Dianoux, F. Volino, H. H. Mol. Phys. 1975, 30(4), 1181-1194.

[80] Jobic, H. J. of Mol. Catalysis A: Chemical 2000, 158, 135-142.

[81] N. Malikova, S. Longeville, J.-M. Zanotti, E. Dubois, V. Marry, P. Turq, J. Ollivier. Phys. Rev. Lett. 2008, 101, 265901.

[82] R. E. Lechner. Solid State Ionics 1995, 7r, 280-286.

[83] V. Marry, B. Rotenberg, P. Turq. Phys. Chem. Chem. Phys. 2008, 10, 4802-4813.

[84] B. Rotenberg, V. Marry, R. Vuilleumier, N. Malikova, C. Simon and P. Turq. Geoch. et Cosmoch. Acta 2007, 71, 5089-5101.

[85] M. Jardat, J.-F. Dufreche, V. Marry, B. Rotenberg and P. Turq. Phys. Chem. Chem. Phys. 2009, 11, 2023-2033.

[86] Teppen, B. J.; Miller, D. M. Soil Sci. Soc. Am. J. 2006, 70(1), 31-40.

[87] Rotenberg, B.; Morel, J.-P.; Marry, V.; Turq, P.; Morel-Desrosiers, N. Geochimica et Cosmochimica Acta 2009.

[88] D. Smith. Langmuir 1998, 14, 5959-5967.

[89] H.J.C. Berendsen, J.R. Grigera, T.P. Straatsma. J.Phys.Chem. 1987, 91, 6269-6271. 